\title{
MARKET CLEARING MECHANISMS FOR EFFICIENTLY INCORPORATING RENEWABLE ENERGY AND MITIGATING CO2
}

\author{
GOLBON ZAKERI AND JAVAD KHAZAEI
}

\section{Abstract}

In recent years there has been a move in the majority of industrialized countries to invest in renewable resources for the production of energy. This move has come about as people worldwide are more aware of negative effects of fossil fuel sources of energy on the environment including the release of green house gases such as CO2. Utilization of renewable sources of energy, for instance harnessing wind power in electricity production, is deemed to be reducing the use of fossil fuels and hence results in the reduction of $\mathrm{CO} 2$. Mechanisms that promote and facilitate utilization of renewable sources of energy are being developed. In particular, recently stochastic programming market clearing mechanisms have been suggested that would seemingly allow for a more efficient use of wind energy hence reduction of fossil fuel use, that ultimately would result in a reduction of CO2. In this paper we will examine the steady state behaviour of participants in an electricity market to fully analyze the hypothesis that the stochastic programming market clearing mechanism is less fossil fuel (and hence CO2) intensive than a conventional two settlement market through some simple examples.

\section{INTRODUCTION}

The United Nations Conference on Environment and Development (UNCED), informally known as the Earth Summit (held in Rio de Janeiro from 3 to 14 June 1992,) produced an international environmental treaty known as the United Nations Framework Convention on Climate Change (UNFCCC). The objective of this treaty as stated in Article 2 of the treaty is "to achieve stabilization of greenhouse gas concentrations in the atmosphere at a level that would prevent dangerous anthropogenic interference with the climate system. Such a level should be achieved within a time-frame sufficient to allow ecosystems to adapt naturally to climate change, to ensure that food production 
is not threatened and to enable economic development to proceed in a sustainable manner."

To achieve the ends set out in the UNFCCC, the Kyoto Protocol was adopted in December 1997 and entered into force in February 2005. As of November 2009, the Protocol has 187 signatories. Under the Protocol the so called Annex I countries, a group of 37 industrialized countries, have committed themselves to the reduction of four green house gases (GHG) that include CO2. It is widely agreed upon that the primary human source of carbon dioxide in the atmosphere is from the use of fossil fuels in energy production (e.g. using coal fired thermal generators,) and transport. Deforestation is also another leading source of $\mathrm{CO} 2$ emissions. Therefore, one potentially substantial way to decrease $\mathrm{CO} 2$ emissions is for countries to disincentivize the use of fossil fuels in the production of energy. To this end, many countries and jurisdictions have imposed, or are about to impose, CO2 related charges (e.g. the European Union, California and New Zealand) and almost all industrialized countries are investing in renewable resources for the production of energy, primarily electricity. Renewable resources such as wind and solar power are volatile as they are dependent on the cooperation of the elements. The wind must blow before the turbines can produce, and it must be sunny in order to utilize solar photovoltaic panels.

This introduces a tradeoff between the volatility of electricity production and emission reduction. To remedy this volatility it is possible to increase the electricity system requirements of dedicated reserve stations (which can be very expensive) or it can be required that volatile renewable generation comes coupled with "firm" production. A third, smarter alternative has been to seek the aid of stochastic programming (see e.g. [8, 2, 11]) to design an economic dispatch mechanism that is hoped to perform more effectively in an uncertain environment. Economic dispatch mechanisms are utilized when clearing the market in electricity pool markets (such as various jurisdictions in the US, Australia, New Zealand, and Scandinavia). We will start with a brief description of the conventional dispatch model used to clear electricity pool markets. In section 3 we present examples that motivate the stochastic programming mechanism for market clearing introduced by Pritchard et al in [8]. We will then review the stochastic programming market clearing mechanism proposed by Pritchard et al.

Introduction of a new market clearing mechanism is likely to result in change of behaviour for the participants in that market. We will examine the effects of the introduction of the stochastic programming 
market clearing mechanism on the behaviour of participants and ultimately on two performance indicators for our market. These indicators are the overall expected welfare and the expected use of thermal resources. While overall expected welfare is a conventional benchmark for the economic performance of a mechanism, in the context of CO2 emissions, the expected use of thermal resources, or the expected use of particular kinds of thermal resources, is a more relevant benchmark. We will provide examples to illustrate that mechanisms designed to encourage harnessing renewable resources can lead to counter-intuitive increase in $\mathrm{CO} 2$ emissions due to the volatile nature of renewable resources such as wind.

\section{REVIEW OF THE CONVENTIONAL ECONOMIC DiSPATCH MODEL}

In this section we briefly review the economic dispatch model that is currently used in wholesale electricity pool markets. Typically in a wholesale electricity market, in each period of the day, each generator offers in generation quantities for each of its plants (possibly located at different grid injection points), at certain prices. In its most general form, the generation offers are supply functions (also known as offer curves) denoted $p=C(q)$, where $C(q)$ is the marginal price of producing quantity $q$. The functions $C(q)$ are required to be increasing. When a generator owns multiple generation units, the efficient generation of a quantity of electricity is achieved by turning on the least costly unit first then moving to more costly units successively (in absence of any constraints). This is the rationale for the requirement that $C(q)$ be an increasing function. It is also the reason why in most electricity spot markets, $C(q)$ is effectively an increasing step function.

The supply offers are collected by the independent system operator (ISO). The ISO also estimates the demand over that period. The ISO then solves a side constrained network optimization problem where the objective is to minimize the total cost of production of electricity. The constraints of this optimization problem reflect that demand must be met at every node of the network, and that physical flow constraints such as transmission line capacities and Kirchhoff's laws must be complied with. Often reactive power modeling is left out of the ISO's dispatch problem and the problem is in fact a direct current equivalent load flow model $[10,9]$. A general model for the ISO's economic dispatch problem (EDP) is formulated below. 


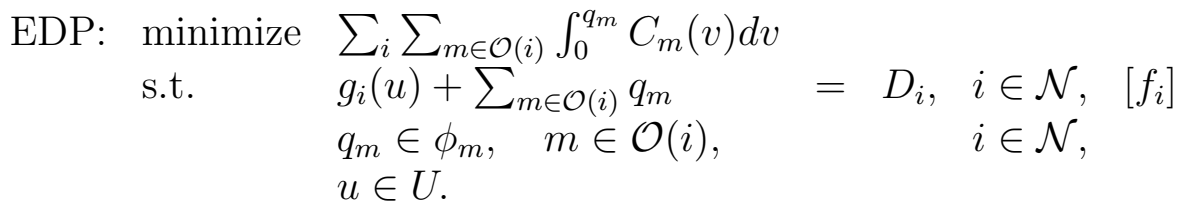

- We use $i$ as the index for the nodes in the transmission grid.

- We use $m$ as the index for the generators.

- $\mathcal{O}(i)$ indicates the set of all generators located at node $i$.

- Generator $m$ can supply quantity $q_{m}$.

- The demand at node $i$ is denoted by $D_{i}$.

- $\phi_{m}$ indicates the capacity of generator $m$.

- The components of vector $v$ measure the dispatch of each generator.

- The components of the vector $u$ measure the flow of power in each transmission line with $u_{i k}$ denoting the flow in the directed line from $i$ to $k$. By convention we assume $i<k$.

- $f_{i}$ denotes the nodal price of electricity for node $i$ and is put in brackets to indicate that it is determined by the optimal duals on the node balance constraints for node $i$. We follow this notation throughout the paper.

Here we require that $u$ lies in the convex set $U$, which means that each component satisfies the thermal limits on each line, and satisfies loop flow constraints that are required by Kirchhoff's Law. The function $g_{i}(u)$ defines the amount of power arriving at node $i$ for a given choice of $u$. This notation enables different loss functions to be modeled. For example, if there are no line losses then we obtain

$$
g_{i}(u)=\sum_{k<i} u_{k i}-\sum_{k>i} u_{i k} .
$$

With quadratic losses we obtain

$$
g_{i}(u)=\sum_{k<i} u_{k i}-\sum_{k>i} u_{i k}-\sum_{k<i} \frac{1}{2} r_{k i} u_{k i}^{2}-\sum_{k>i} \frac{1}{2} r_{i k} u_{i k}^{2} .
$$

As indicated above, one of the functions of the ISO is to set the price. The price of electricity is determined as the shadow price $f_{i}$ of the node balance constraints above that indicate demand must be met at all nodes. This price is the system cost of meeting one more unit of demand at node $i$. This method of determining the electricity price is sometimes referred to as locational marginal pricing (LMP). New Zealand and the PJM market in the US are examples of electricity markets with LMP. 
In some electricity markets, such as the England and Wales market, all trading takes place at a single node and the market runs as a single node market. Clearly this is also the case if the network constraints are inactive, e.g. in periods where the electricity flow is less than the capacity of the line that it flows on, for every line, and when the losses are negligible enough to ignore. In such situation, the optimization problem EDP reduces to the following single node economic dispatch problem (SNEDP).

$$
\begin{array}{lll}
\text { SNEDP: } & \text { minimize } & \sum_{m} \int_{0}^{q_{m}} C_{m}(v) d v \\
\text { s.t. } & \sum_{m} q_{m} \\
& & q_{m} \in \phi_{m},
\end{array}, \quad[f]
$$

Here $D$ denotes the total demand and $f$ is the clearing price of electricity.

As alluded to earlier, frequently the supply functions are specified as offer stacks. Offer stacks are increasing piecewise constant functions where each piece, often called a tranche, is specified by a quantity and a price. Let $a_{t}$ and $\phi_{t}$ denote the price and the quantity of tranche $t$ respectively. Then the optimization problem SNEDP reduces to simply ordering the available tranches of energy in an increasing order then observing where the total demand $D$ meets this aggregated supply function as depicted in Figure 3.1.

For the rest of this paper, we confine our attention to the case of the single node electricity markets. Although the results presented in this paper stand when we have an electricity market over a network (including losses etc,) it is clearer to see the effects of uncertainty in a single node case. We also bypass anomalies that are introduced through the transmission network in this paper. It is worth noting that Downward [4] examines what can happen to emissions when a CO2 charge is introduced into an electricity market with a transmission network. Downward starts by proving that when generators offer their production at their true marginal cost, then introducing a CO2 charge will result in the reduction of emissions. However, when exercise of market power is present, then even in a simple two node network electricity market, with a single transmission line, there are situations in which the introduction of a CO2 charge can in fact lead to an increase of production of electricity and hence increased emissions. This is because the generators end up with a different cost structure (that now have the embedded CO2 charges,) and their behaviour is different. In Downwrad's model the introduction of the CO2 charge results in closer marginal costs for the generators which induces more competition and production in the market. Ultimately, this results in increased CO2 


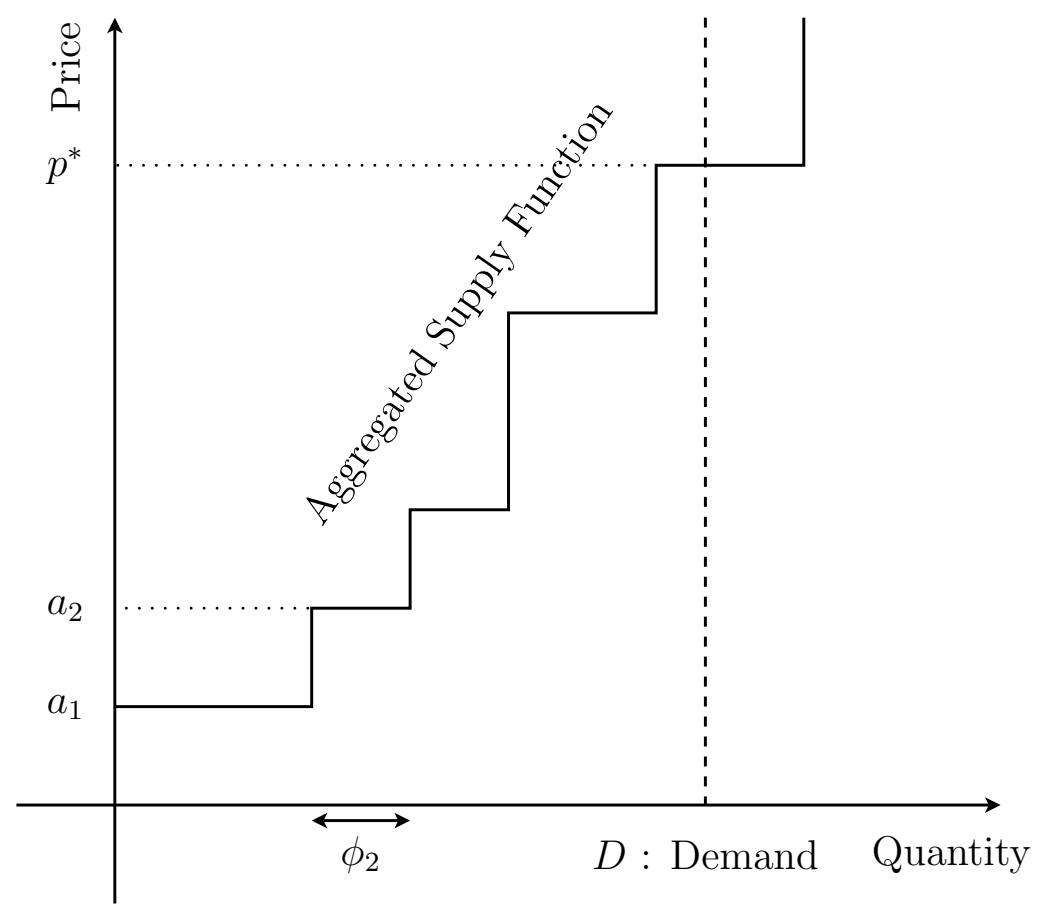

FIGURE 3.1. The simple one-node conventional market clearing mechanism

emissions. This is an example of the general theory of second best that manifests in economics literature frequently dating back to 1950s [6]. Here the introduction of an 'improvement' actually leads to line constraints binding which itself leads to a worse market outcome in steady state. We examine a similar phenomenon in relation to introduction of the stochastic programming market clearing mechanism.

\section{Review of the STOChastic PROgRAmming Market CLEARING MECHANISM}

In this section, we introduce a stochastic programming economic dispatch model. The central idea here is to view the dispatch problem as having two stages, following the conventional models of two stage stochastic programs [1].

Currently, in wholesale electricity markets such as the NZEM, generators submit their supply functions to the ISO a number of periods prior to the trading period the offers are intended for (in some markets the offers are submitted an entire day ahead of the actual trading period). Prior to the start of the trading period in question, the 
ISO solves the conventional dispatch problem EDP using the submitted offers and estimates of demand (for instance expected demand) and proceeds to dispatch generation according to that plan from various generators. During the trading period, various random variables (such as wind farm output and demand) are realized and there will be discrepancies between the planned dispatch of generation, the realized generation (from the wind farms for instance,) and actual consumption. Currently, ISOs rely on a dedicated frequency keeping, load following, station to close this difference (at least in short sub-intervals of the trading period prior to a re-dispatch). With the increased volatility introduced through intermittent renewable generation such as wind, this system is unlikely to be sufficient, and it will almost certainly not function efficiently. This will hinder the current policies that preferentially dispatch wind over other forms of generation.

In the stochastic programming mechanism, in stage one, an initial dispatch is computed in advance, with only probabilistic estimates for quantities such as wind generation. This first stage produces a plan of production, which will be modified once the random variables (such as quantity of energy produced from wind) are realized. The second stage then represents "real time" dispatch, i.e. the actual dispatches over a short period. (Pritchard et al. intend the duration of this second stage to be somewhere between a minute and an hour, so that it would coincide with a trading period or be a sub-interval of that period). During this second stage, outputs of wind farms take on realizations unknown in the first stage. Adapting to these changes will require modifications to the initial dispatch plan. Therefore, various generators may have to ramp up or down. The formulation of the stochastic program will enable all firms to bid in what are termed "regulations". Through regulation bids, firms indicate whether or not they have the ability to deviate production from the level that was agreed upon in the first stage and how much would this deviation cost. The deviations can be above or below the levels indicated in the first stage production plan therefore the regulation costs are for both up or down deviations when such deviations are possible. The effect of enabling such bids is that the market is opened to all available participants and not restricted to certain designated load following stations. Therefore, it is hoped that through this increased participation, the system can cope with more volatility. Furthermore, as the stochastic programming market clearing mechanism produces the dispatches taking account of the distribution of volatility (for instance in wind production,) it will be a more efficient dispatch over a deterministic dispatch model, provided the market participants bid in the same offer curves in both mechanisms. 
In the stochastic programming mechanism, the optimization problem aims to minimize expected total cost of generation and regulation in the real time. In addition to the first stage decisions, the stochastic program gives a plan for each of the possible states of uncertainty i.e. scenarios. A general model for the stochastic programming dispatch problem is as follows.

$$
\begin{array}{llr}
\text { SNSP: } & & \\
\min & \sum_{s} \theta_{s} \sum_{m}\left(\int_{0}^{q_{m}+x_{m, s}} C_{m}(v) d v+\int_{0}^{x_{m, s}} \rho_{m}(v) d v\right) & \\
\text { s.t. } & \sum_{m} q_{m}=Q, & s \in S, \quad\left[\theta_{s} p_{s}\right] \\
& Q+\sum_{m} x_{m, s}=D_{s}, &
\end{array}
$$

Here we use the same notation as SNEDP for the common parameters between this model and SNEDP. In this model, we have assumed a finite discrete distribution where each scenario is indexed by $s$. The probability associated with scenario $s$ is denoted $\theta_{s}$. Let $q_{m}$ denote the first stage production quantity of generator $m$; we use $x_{m, s}$ as the deviation in the spot market from $q_{m}$ if scenario $s$ occurs (this can be positive or negative). The components of vector $v$ measure the dispatch of each generator, and $C_{m}$ and $\rho_{m}$ indicate the marginal generation and deviation cost functions of generator $m$ respectively. Note that in this market clearing mechanism, $C_{m}$ and $\rho_{m}$ are offered by the generators participating in the market.

Similar to the conventional economic dispatch, SNSP is responsible for determining the nodal prices. The first stage price is determined as the shadow price of the constraint that determines the total generation in the first stage $(f)$. The dual variables $p_{s}$, of the constraints that enforce the total generation should meet the demand in the spot market, determine the probability included price of the second stage in scenario $s$. The payment to generator $m$ if scenario $s$ happens is equal to $f q_{m}+p_{s} x_{m, s}$. In what follows, we will discuss a simple illustrative example that demonstrates the desired properties of the stochastic programming market clearing mechanism, which are the reasons why they have been advocated in the literature.

Example 1. Consider a simple electricity market over a single node in which there exists a cheap non-thermal (for instance nuclear) and an expensive thermal generator. These generators are labeled $G 1$ and $G 2$ respectively and the production cost of $G 1$ is assumed to be $\$ 20.00$ per MW and that of $G 2$ is assumed to be $\$ 27.00$ per MW. We further assume that our cheap generator $G 1$ is not easily able to generate more than its pre-dispatch quantity in the spot market. Ramping up or down 
in spot is comparatively costly for $G 1$. In contrast, we assume that our thermal generator is flexible and can change its output at a significantly lower cost than $G 1$ (at $\$ 10.00$ versus $\$ 2.00$ for $G 2$ ). The generation and ramp costs of these generators are noted in Figure 4.1 along with generator capacities (both fixed at $\$ 5.00 \mathrm{MW}$ ) and demand. We have assumed that demand is deterministic and fixed at $4 \mathrm{MW}$. Here the conventional deterministic market clearing will result in a dispatch of 4 MWs from $G 1$ and nothing from $G 2$. Total thermal generation is zero in this case.

Now imagine a wind generator is added to the system. This wind generator can produce nothing or 2 units with the probabilities 0.6 and 0.4 respectively. Figure 4.2 illustrates this situation.

Consider a market clearing mechanism that dispatches wind generator in all the scenarios (this is following the current New Zealand dispatch policy that prefers the dispatch of wind). Therefore, this example can be represented as figure 4.3 with wind generation taken into account as offsetting demand. As discussed above, the conventional dispatch system solves a pre-dispatch problem for the expected demand (i.e. $3.2 \mathrm{MW}$ ). In this case, the pre-dispatch quantity of the nuclear and thermal generators are $3 \mathrm{MW}$ and $0 \mathrm{MW}$ respectively. When wind generation realizes, the ISO will have to re-dispatch generation. For example, if scenario 2 happens and wind does not blow, 1 more unit is produced and thermal generator $G 2$ is dispatched for this amount, as ramp up is expensive for generator G1. Table 1 contains the dispatch results for this example when the conventional market clearing mechanism is used.

The same problem illustrated in figure 4.2, can be solved using the stochastic programming market clearing mechanism. In this case, the stochastic programming market clearing mechanism pre-dispatches $G 1$ (the cheaper and cleaner but less flexible generator) 4MW. G2 is not pre-dispatched. The second stage decisions in the stochastic programming market clearing determine the adjustments that are needed in each scenario once the volume of generation from wind is realized. Table 2 contains the stochastic programming dispatches along with the corresponding costs. Note that from Tables 1 and 2 it is evident that the stochastic programming approach is more efficient, i.e. it has a lower expected cost. Furthermore, the thermal dispatch is less in the market that is cleared through stochastic programming as compared to the market cleared from the deterministic approach. Both attributes are desirable and reasons for why stochastic programming has been proposed as a mechanism for clearing electricity pool markets involving uncertainty. Nevertheless, introduction of a new mechanism can 


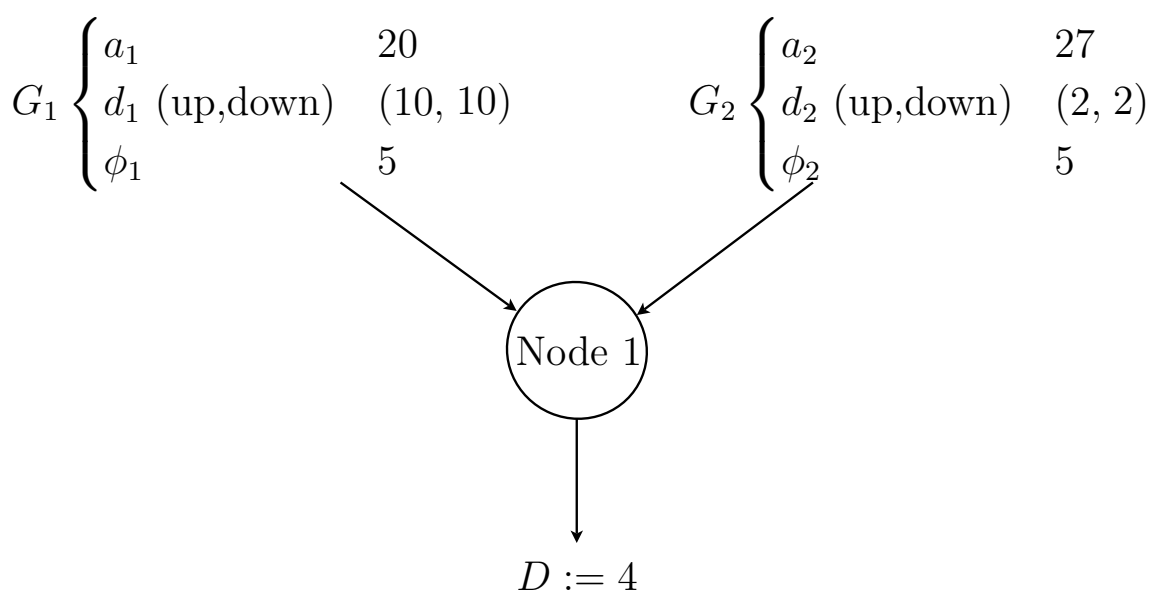

FiguRE 4.1. One-node example market structure

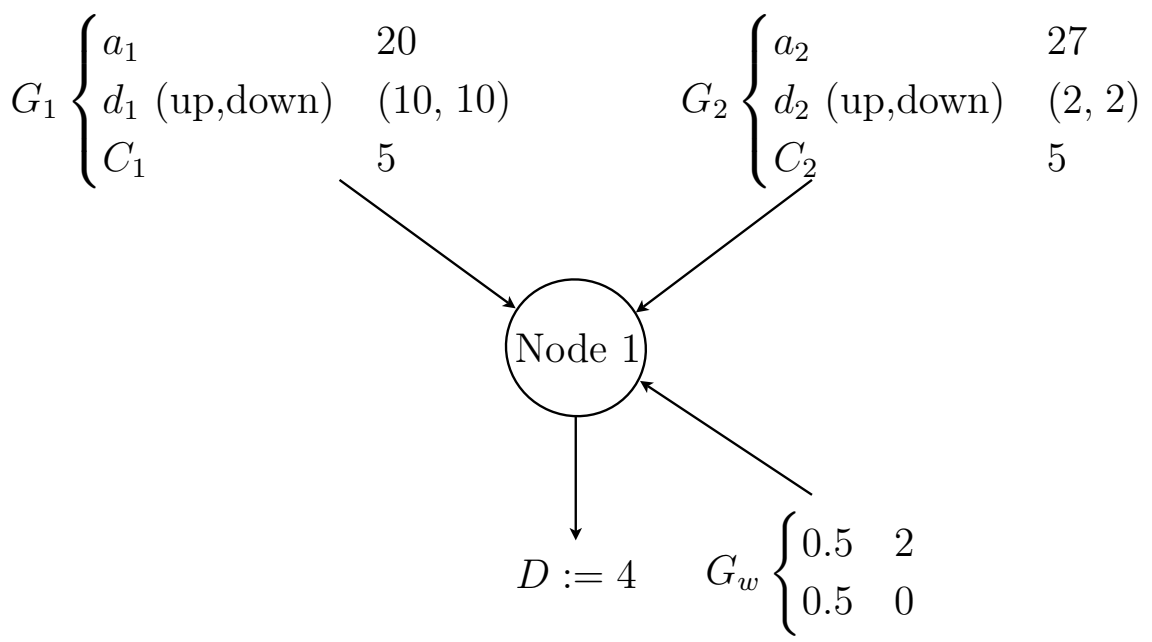

Figure 4.2. Market structure after introducing a new wind plant to the electricity market

change the behaviour of the participants in a market, and we will turn our attention to this issue in the next section. 


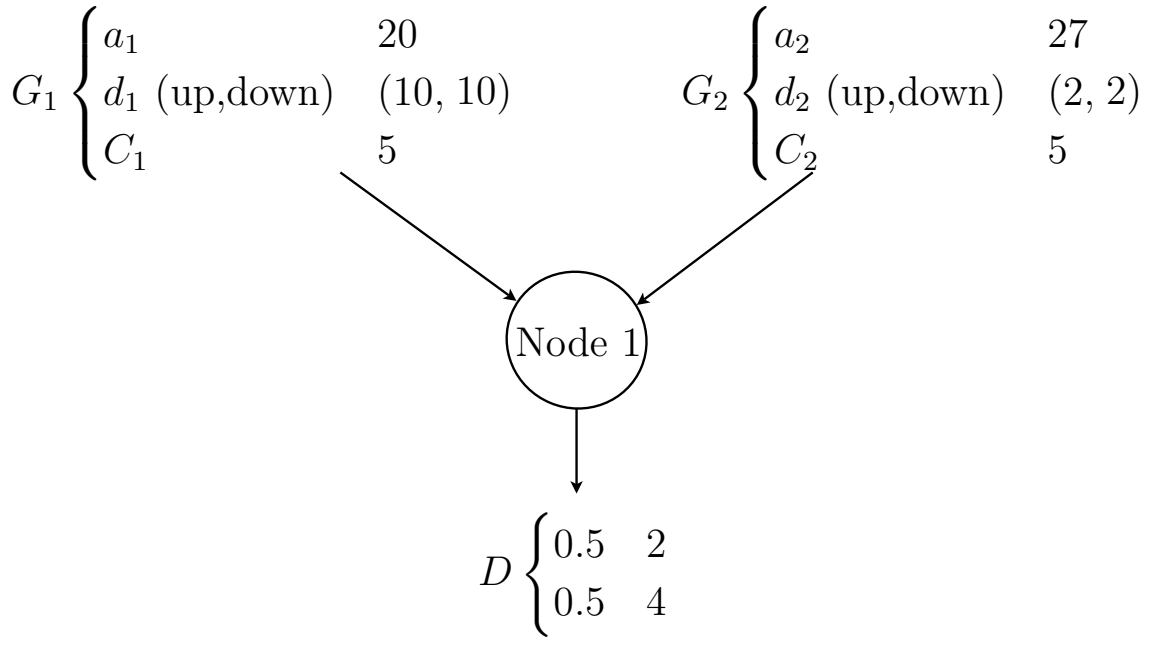

FIGURE 4.3. The equivalent system, after wind introduction

\begin{tabular}{|r|r|r|r|r|}
\hline & Pre-dispatch & Scenario 1 & Scenario 2 & Expected Value \\
\hline \hline$G_{1}$ & 3 & 3 & 2 & 2.6 \\
\hline$G_{2}$ & 0 & 1 & 0 & 0.6 \\
\hline Cost & 60 & 89 & 50 & 73.4 \\
\hline Thermal Use & 0 & 1 & 0 & 0.6 \\
\hline
\end{tabular}

TABLE 1. Generation quantities and total cost of the conventional market for example 1

\begin{tabular}{|r|r|r|r|r|}
\hline & Pre-dispatch & Scenario 1 & Scenario 2 & Expected Value \\
\hline \hline$G_{1}$ & 4 & 4 & 2 & 3.2 \\
\hline$G_{2}$ & 0 & 0 & 0 & 0 \\
\hline Cost & 80 & 80 & 60 & 72 \\
\hline Thermal Use & 0 & 0 & 0 & 0 \\
\hline
\end{tabular}

TABLE 2. Generation quantities and total cost of the stochastic programming market for example 1

\section{IMPACTS OF THE INTRODUCTION OF STOCHASTIC PROGRAMMING MARKET CLEARING MECHANISM IN PRESENCE OF MARKET POWER}

While dispatches acquired through a stochastic programming market clearing mechanism are more efficient than dispatches that are arrived 
at from the conventional deterministic EDP, the change in the market clearing mechanism may affect the offer behaviour of the market participants. The efficiency gains of the stochastic program over the conventional deterministic EDP are only proven when the offers into both market clearing problems are the same. In order to make the right efficiency comparisons, we must account for possible changes in the offer behaviour of the participants as well. To capture the change in the offer behaviour of participants, in response to a change in the market clearing mechanism, we appeal to the concept of Nash equilibria for describing a steady state behaviour of the participants under different mechanisms. The Nash equilibrium was introduced by Antoine Augustin Cournot in 1883 [3], and subsequently formalized by John Forbes Nash in 1951 [7]. It is defined as the state of the game, given by various players' strategies, where no player has incentive to change his/her strategy given that all other players' strategies remain fixed.

In the following example, we look at another stylized electricity market over a single node. We allow for demand to be price responsive, and we use two scenarios of demand, that similar to our previous examples could correspond to the introduction of an uncertain amount of generation due to wind. We are interested in examining the steady state offer behaviour of the generators when the market clears through a stochastic program as compared to when the market clears in two different settlements, one before and one after the realization of (demand) uncertainty. To make the model tractable, so we can compute equilibria, we restrict our generators to offer linear supply functions into the two settlement market. Here they submit a linear supply function offer stack once, and this same stack is used for any re-dispatch purposes (much like what currently happens in New Zealand). In the stochastic programming case, in addition to the linear supply function, we allow our generators to offer in a deviation cost parameter too. All offers are indicated at the start, and the stochastic program solves for dispatch knowing these parameters.

Let us represent the total amount of generation from generator $i$ in scenario $s$ by $y_{i, s}$ and deviation in the spot market by $x_{i, s}$. The quadratic cost function $\alpha y_{i, s}+\beta y_{i, s}^{2}$ determines generator $i$ 's cost of generation in scenario $s$, and $\delta x_{i, s}^{2}$ shows his cost of deviation, in this scenario.

Example 2. Consider a market with two symmetric thermal generators with marginal cost of generation equal to $30+q$. Also, assume that the cost of deviation in the spot market is zero. Two equally 
TABLE 3. Social welfare and thermal generation in example 2

\begin{tabular}{|c|c|c|}
\hline & Social welfare & $\begin{array}{c}\text { Expected thermal generation } \\
\text { from each generator }\end{array}$ \\
\hline \hline Stochastic programming & 3210.59 & 31.6561 \\
\hline Two settlement mechanism & 3122.81 & 26.2574 \\
\hline
\end{tabular}

likely states of demand uncertainty exist, which affect the y-intercept of demand function. Demand is elastic and $p_{s}=Y_{s}-D$ determines the relationship between spot price $\left(p_{s}\right)$ and demand quantity $(D)$. Also assume $Y_{1}=100$ and $Y_{2}=150$. Figure 5.1 illustrates this market. Note that $G(\alpha, \beta, \delta)$ determines cost parameters introduced earlier.

If we compute the equilibrium quantities of this market under stochastic programming and the conventional two settlement mechanism, we end up with two different sets of offers. Table 3, shows that while stochastic programming leads to higher expected social welfare, it also increases expected total generation from thermal resources. This increase in the expected total generation from thermal resources then implies an increase in the CO2 emissions. This is an unintended consequence of introduction of an intermittent renewable resource such as wind coupled with a market clearing mechanism (the stochastic program,) that was designed to utilize wind more efficiently. This example serves to warn that change of behaviour of participants in response to a change in market clearing mechanism must be taken into account.

We have demonstrated this concept on a small example. The problems that arise as best response problems of individual generators for the above game, under the stochastic programming market clearing mechanism, are non-convex. Even though the size of these problems is relatively small, we have to solve many of these non-convex problems to global optimality before we reach an equilibrium. This process is time and computation intensive and at this stage prohibits solving large examples. Below we outline the full extent of the computational procedure that provides the equilibria of this example.

5.1. Computation of a Nash equilibrium. For the above example, we have presented Nash equilibria under two different market clearing mechanisms. There are different ways of computing the Nash equilibrium of a game. One approach is to derive conditions for the best response of each player, given all other players' strategies, then find strategies where all of these conditions are satisfied simultaneously. The best response function of a participant determines that player's 


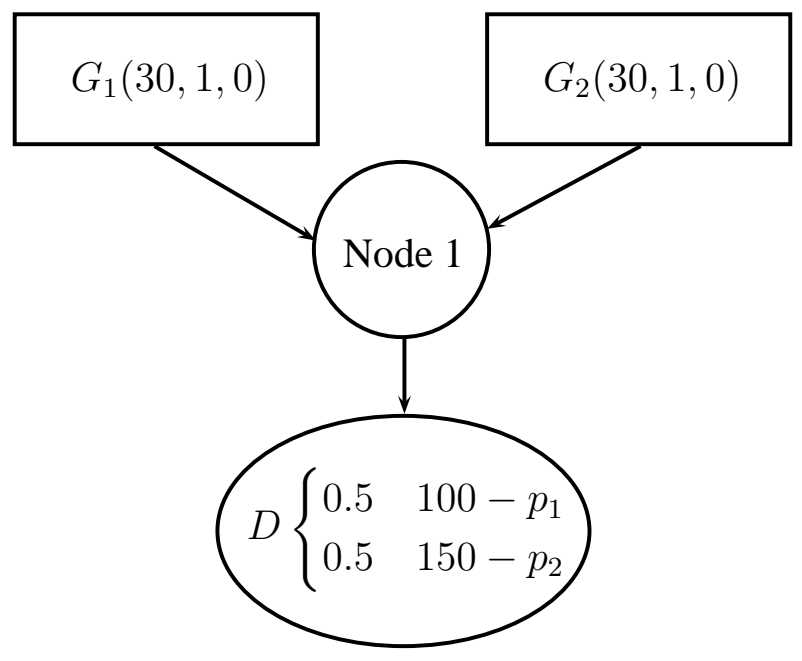

FiguRE 5.1. generators' cost parameters and demand scenarios for example 2

best strategy as a function of the other players' strategies (as fixed parameters).

For our example, for an electricity market with the stochastic programming clearing mechanism, player $i$ solves the following optimization problem as his/her best response.

$$
\begin{aligned}
\operatorname{BR}[i]: \max _{C_{i}, \rho_{i}} \pi_{i}= & f q_{i}+\sum_{s} \theta_{s}\left(p_{s} x_{i, s}-\alpha_{i}\left(q_{i}+x_{i, s}\right)-\frac{\beta_{i}}{2}\left(q_{i}+x_{i, s}\right)^{2}\right. \\
& \left.-\frac{\delta_{i}}{2} x_{i, s}^{2}\right) \\
\text { s.t. } \quad & \{\boldsymbol{q}, \boldsymbol{x}, \boldsymbol{f}, \boldsymbol{p}\} \text { are optimal for SNSP } \\
& \left\{C_{i}, \rho_{i}\right\} \in \Psi_{i}
\end{aligned}
$$

Here

- $\pi_{i}$ stands for the expected profit function of firm $i$ which is the difference between its expected income and its expected cost of production.

- $\Psi_{i}$ indicates the constraints imposed by the regulator on offered marginal generation and deviation cost functions of player $i$ i.e. $C_{i}$ and $\rho_{i}$.

To find the equilibrium of this game, by simultaneously solving all $\operatorname{BR}[i](\forall i)$, we need to be able to derive a set of equations equivalent to each optimization problem $\mathrm{BR}[i]$. Although it is tempting to replace 
$\mathrm{BR}[i]$ by its KKT conditions, note that $\mathrm{BR}[i]$ is not a convex optimization problem, due to the presence of some bilinear terms in the objective (such as $f q_{i}$ ) and quadratic terms in the constraints. Therefore, the KKT conditions are not equivalent to the optimization.

In order to find an equilibrium for the above game we use fictitious play. The process we use is as follows.

(1) The process starts from one (possibly random) strategy for each player.

(2) While any player (e.g. $j$ ) has incentive to deviate from its current strategy,

(a) Solve $\operatorname{BR}[j]$.

(b) Update the current strategy vector with the solution of $\mathrm{BR}[j]$.

(3) The resulting strategy vector is the equilibrium of the game, based on the definition of Nash equilibrium.

As we have already mentioned, the optimization problem $\operatorname{BR}[i]$ is not a convex optimization problem. This means that it is necessary to use global optimization to solve this problem. For solving this optimization problem, we have used the global solver of LINGO. The global solver of LINGO guarantees the optimality of its final solution using a branch and bound approach. Here a sequence of piecewise convex relaxations of the original (non-convex) problem are solved. The convex relaxations are derived using bounds on the variables. If the optimal solution of the relaxed problem is feasible for the original problem, it is also the optimal point of the original problem. If not, further enhancement is made through dividing up the domain of the objective function and creating more accurate, piecewise convex functions on each part of the domain. The process of branching continues until are branches end

with an optimal point. Note that user defined tolerances on slitting procedure make this method a finite process. For more information about the mathematics behind this global solver see [5]. The tolerance that we have used as the minimum acceptable difference between best response strategies of firms in different turns to continue the process is of order of $10^{-10}$.

\section{Conclusion}

In this article, we have discussed two market clearing mechanisms for settling electricity pool markets that involve uncertainty. The conventional approach is to rely on forecasts or expected values of random variables such as output of a wind farm, and plan dispatches. Only once the uncertainty is resolved does the system operator look for making 
changes to the plan intact and this may be very costly. In contrast, a smarter new mechanism has been introduced that takes into account the distribution of uncertainty in the future and makes plans with this distribution in mind. The latter mechanism is provably more efficient and desirable if the inputs (i.e. the generator offers and demands,) are the same for both models. It should be kept in mind that changing a market clearing mechanism will likely change the offer behaviour of participants. When we closely examine this premise in the context of an example, we find that counter-intuitively the change over to the smarter stochastic programming market clearing can create undesirable effects. Such examples further verify that in markets where the exercise of market power is not ruled out, it is possible that a seeming improvement can lead to worsening of outcomes.

\section{REFERENCES}

[1] J. R. Birge and F. Louveaux. Introduction to Stochastic Programming. Springer, New York, 1997.

[2] F. Bouffard, F. D. Galiana, and A. J. Conejo. Market Clearing with Stochastic Security. IEEE Transactions on Power Systems, 20:1818-1826, 2005.

[3] A. Cournot. Researches into the Mathematical Principles of the Theory of Wealth, originally published in French, translated (1897) by Nathaniel Bacon. New York: MacMillan, 1838.

[4] A. Downward. Carbon charges in electricity systems may increase emissions. Energy Journal, To appear.

[5] Y. Lin and L. Schrage. The global solver in the LINDO API. Optimization Methods and Software, 24(4):657-668, 2009.

[6] R. G. Lipseyi and K. Lancaster. The General Theory of Second Best. The Review of Economics Studies, 24:11-32, 1956.

[7] J. F Nash. Equilibrium points in n-person games. Proceedings of the National Academy of Sciences of the United States of America, 36(1):48-49, 1950.

[8] G. Pritchard, G. Zakeri, and A. Philpott. A single-settlement, energy-only electric power market for unpredictable and intermittent participants. Operations Research, 58:1210-1219, 2010.

[9] Oren S. S., Spiller P. T., Varaiya P., and Wu F. Nodal Prices and Transmission Rights: A Critical Appraisal. Electricity Journal, 8(3):24-35, 1995.

[10] F. Schweppe, M. Caramanis, R. Tabors, and R. Bohn. Market Operations in Electric Power Systems. Kluwer, Boston, 1988.

[11] S. Wong and J. D. Fuller. Pricing Energy and Reserve Using Stochastic Optimization in an Alternative Electricity Market. IEEE Transactions on Power Systems, 22:631-638, 2007. 